\title{
Modelagem matemática do fluxo de emergência de plantas daninhas: ferramenta para decisão no manejo de cultivos
}

\author{
Mathematical modeling of the drought emergency flow: \\ crop management decision tool
}

\section{Renan Ricardo Zandoná1*, Dirceu Agostinetto², Queli Ruchel²}

Resumo - O surgimento de biótipos de plantas daninhas resistentes a herbicida e o aumento da pressão social para reduzir sua utilização, criou a necessidade de desenvolver ferramentas para aperfeiçoar a aplicação de herbicidas e melhorar o controle de plantas daninhas. Desta forma, o desenvolvimento de modelos de emergência de plantas daninhas tornou-se crucial para o desenvolvimento de estratégias de gerenciamento em áreas agrícolas. Esta revisão tem por objetivo apresentar os fatores que influenciam o fluxo de emergência de plantas daninhas, os principais modelos utilizados, os procedimentos da modelagem e os impactos práticos de sua utilização no manejo. O fluxo de emergência das plantas daninhas correlaciona-se diretamente com padrões de resposta das sementes à interferência dos fatores ambientais e práticas culturais. Os modelos de emergência empíricos e mecanicistas desenvolvidos permitem prever a emergência de plantas daninhas em diferentes anos e regiões geográficas, baseados em condições climáticas e ecofisiológicas. Os modelos mecanicistas são mais complexos e permitem a melhor compreensão sobre a emergência de plantas daninhas, enquanto modelos empíricos apresentam a simplicidade e a flexibilidade necessária para a tomada de decisão de controle. O conhecimento do fluxo de emergência de plantas daninhas permite desenvolver estratégias de manejo que proporcionam maior habilidade competitiva à cultura e, em decorrência, menores perdas de produtividade; bem como a seleção de medidas de controle que diminuam a utilização de herbicidas, a contaminação ambiental e aprimorem as tecnologias utilizadas na agricultura.

Palavras-chave: modelo empírico, gerenciamento, tempo hidrotérmico

Abstract - The appearance of weed biotypes resistant to herbicides and the increase in social pressure to reduce its use, created the need to develop tools to refine herbicides application and improve weed control. In this way, the development of weed emergence models has become crucial for the development of management strategies in agricultural areas. The objective of this review is to show the factors that influence the emergence flow and the main models used to predict weed emergence, the procedures of modeling and the practical impacts of their use for weed management. The emergence flow of weeds correlates directly with seed response patterns to the interference of environmental factors and cultural practices. The developed empirical and mechanistic emergence models allow predict weeds emergence in different years and geographic regions, based on climatic and ecophysiological conditions. However, mechanistic models are more complex and allow better

Recebido: Abril 18, 2017. Aceito: Maio 11, 2017.

${ }^{1}$ Programa de Pós-graduação em Fitossanidade, Departamento de Fitossanidade, Faculdade de Agronomia Eliseu Maciel, Universidade Federal de Pelotas - UFPel, Campus Universitario, Capão do Leão, CP 354, CEP 96010-900, Pelotas, RS, Brasil. E-mail: renan_zandona@hotmail.com

${ }^{2}$ Universidade Federal de Pelotas - UFPel, Pelotas, RS, Brasil 
comprehension of weed emergence, but empirical models provide the simplicity and the flexibility necessary for decision making. The knowledge of weed emergence flows allow the development of management strategies that provide a greater competitive ability to the crop and reduce productivity losses, as well as the selection of control measures that reduce the use of herbicides, environmental contamination and improve technologies used in agriculture.

Keywords: empirical model, management, hydrothermal time

\section{Introdução}

$\mathrm{Na}$ agricultura a tecnologia é utilizada para planejar, monitorar e executar atividades fitossanitárias no campo durante o desenvolvimento das culturas, visando melhorar o controle de plantas daninhas, minimizando os custos de produção. O conhecimento dos sistemas biológicos onde estas tecnologias são empregadas torna-se fundamental para a implementação de estratégias de manejo (Werle et al., 2014b) e aprimoramento de tecnologias.

O manejo de plantas daninhas depende quase que exclusivamente da aplicação de herbicidas; entretanto, a expansão da agricultura orgânica, os problemas de plantas daninhas resistentes a herbicidas, a diminuição de moléculas disponíveis no mercado e a maior adoção do manejo integrado de plantas daninhas (MIPD), tendem a alterar esse panorama.

As maiores limitações para implementação do MIPD é a falta de conhecimento sobre a biologia e ecologia das espécies (Sadeghloo et al., 2013). $\mathrm{O}$ conhecimento de aspectos relacionados à germinação como temperatura ótima, causas da dormência e velocidade de emergência, auxiliam na adoção de práticas adequadas de manejo de culturas (Guo e Al-Khatib, 2003). A época e o tempo de germinação das sementes de plantas daninhas estão relacionados ao sucesso da espécie no agroecossistema (Koger et al., 2004), determinando a capacidade competitiva com vizinhos, sobrevivência a estresses e taxa de reprodução. Dessa forma, o desenvolvimento de modelos de emergência torna-se crucial para aprimoramento de estratégias de manejo de plantas daninhas.

Estudo e levantamento de populações de plantas daninhas em ambientes agrícolas, além de permitirem a identificação da flora infestante e sua evolução na área, podem ter aplicação na predição de infestações em culturas agrícolas (Voll et al., 1997a). Taxas de emergência de espécies do banco de sementes podem servir para adequar o manejo, para racionalização do uso de herbicidas (Voll et al., 1997b). Ainda segundo os autores, a emergência de espécies daninhas em pré-semeadura da cultura pode ser eliminada por práticas de manejo, reduzindo a infestação e a sobrevivência da espécie.

Dessa forma, esta revisão teve por objetivos apresentar os fatores que influenciam o fluxo de emergência de plantas daninhas, modelos matemáticos empíricos e mecanicistas para prever a emergência destas espécies, procedimentos da modelagem mais utilizados e impactos práticos sobre o manejo com a informação destes estudos.

\section{Fatores ambientais que influenciam o fluxo de emergência}

O fluxo de emergência das plantas é definido como a dinâmica de emergência da infestação para determinada espécie em função do tempo, sendo influenciado pelas condições edafoclimáticas, manejo do solo e aplicação de herbicidas (Blanco, 2014). Observações realizadas a campo demonstraram que o principal fluxo de emergência apresenta tendência de ocorrer em determinados períodos do ano, variável em função de cada espécie (Blanco e Blanco, 1991). 
O fluxo de emergência das plantas daninhas correlaciona-se diretamente com padrões de resposta das sementes à interferência dos fatores ambientais predominantes e práticas culturais. A germinação das sementes e emergência de plantas pode ser afetada por fatores intrínsecos, como o estádio de maturação, dormência e longevidade (Martins et al., 2000) e/ou ambientais, como a disponibilidade de água, temperatura, luz, oxigênio, $\mathrm{pH}$, salinidade do solo e profundidade de enterrio (Chauhan et al., 2006a).

Todos estes fatores têm o potencial de influenciar o comportamento de sementes de plantas daninhas, contudo cada espécie exige requisitos específicos quanto à disponibilidade de água, temperatura e luz para a ocorrência dos processos germinativos. A falta de algum desses fatores pode fazer com que a germinação ocorra de forma lenta e em menor escala, ou ainda, induzir à dormência (Canossa et al., 2008). Temperatura e luz são considerados os sinais ambientais que regulam a germinação, distribuição das espécies e interações ecológicas (Ali et al., 2013), quando a umidade do solo é suficiente para embebição da semente, pois a água faz parte das estruturas moleculares como proteínas e ácidos nucleicos, bem como de lipídeos e carboidratos (Guimarães et al., 2008).

A temperatura afeta tanto a porcentagem, quanto a velocidade de germinação das sementes, estando relacionada com as reações bioquímicas necessárias para o início do processo germinativo (Carvalho e Nakagawa, 2000). As sementes respondem a temperatura dentro de uma faixa relacionada à região de origem geográfica da espécie (Andrade et al., 2000), sendo estas consideradas as temperaturas de máxima e mínima, caracterizam pontos críticos onde dificilmente ocorre germinação (Derakhshan et al., 2014). Nesta faixa, existe a temperatura ótima, na qual ocorre máxima germinação, como em menor intervalo de tempo (Popinigis, 1985), variável entre as espécies. Além disso, algumas espécies necessitam de temperaturas constantes para a germinação, como Conyza bonariensis (L.) e Conyza canadensis
(L.) (Vidal et al., 2007; Yamashita e Guimarães, 2011), Alternanthera tenella (L.) (Canossa et al., 2008), Mimosa pudica (L.) e Ipomoea asarifolia (Ders.) (Souza Filho et al., 2001) e, para outras a alternância de temperatura favorece esse processo Digitaria bicornis (Lam.) e D. ciliaris (Retz.), D. horizontalis (Willd.) e D. insularis (L.) (Mondo et al., 2010).

A luz é outro fator fundamental para a germinação das sementes, pois induz a expressão de genes que iniciam os promotores da germinação por sinais liberados pelo fitocromo, estando envolvida na regulação do crescimento e desenvolvimento de plântulas. É necessária para a germinação de muitas espécies de plantas daninhas (Canossa et al., 2008), sendo essa resposta denominada de fotoblástica. Deste modo, existem sementes que germinam apenas sob rápida exposição à luz ou após longo período de exposição, podendo-se citar as espécies Bidens pilosa (L.), Acanthospermum sp. (L.), Xanthium sp. (L.), Galinsoga sp. (L.), Eupatorium sp (L.), D. horizontalis (Willd.) (Klein e Felippe 1991), C. bonariensis (L.) e C. canadensies (L.) (Vidal et al., 2007).

As sementes cuja germinação é desencadeada no escuro são consideradas fotoblásticas negativas, como as espécies de Ipomoea grandifolia (Dammer), I. nil (L.), Merremia aegyptia (Orzari et al., 2013), D. ciliaris (Retz.) e D. insularis (L.) (Mondo et al., 2010). Há outras que não respondem à luz, sendo estas fotoblásticas neutras, pois germinam na presença ou ausência de luz como Euphorbia heterophylla (L.), Sida glaziovii (L.) e Brachiaria plantaginea (L.) (Salvador et al., 2007; Vázquez-Yanes e Orozco-Segovia, 1990). Dessa forma, a intensidade, o comprimento de onda e o fotoperíodo regulam o início da germinação, pois o fitocromo interpreta e traduz sinais luminosos para a expressão dos genes que iniciam os promotores da germinação.

Além dos fatores citados, a concentração de oxigênio é importante para a germinação, visto que grande parte das espécies não apresenta capacidade de germinar sob condição de hipoxia. Então, a temperatura do solo, luz, disponibilidade de água e 
composição do ar (oxigênio, dióxido de carbono) são essenciais para o início e continuidade do crescimento de espécies vegetais, particularmente em relação à germinação e emergência das plantas (Calado et al., 2011). Assim, a partir de informações climáticas, pode-se inferir qual o nível de risco de infestações de plantas daninhas nas áreas agrícolas.

\section{Modelos empíricos e mecanicistas}

Pesquisadores desenvolveram modelos matemáticos empíricos e mecanicistas para prever a emergência de espécies de plantas daninhas. Esses modelos são desenvolvidos com base em condições ambientais, o que permite o uso para prever a emergência de plantas daninhas em diferentes anos e regiões geográficas (Grundy, 2003; Werle et al., 2014a).

Os modelos empíricos têm sido utilizados para prever a emergência das plantas daninhas baseados no tempo térmico (TT) ou tempo hidrotérmico (TH) (Forcela, 2000; Myers et al., 2004; Chauhan et al., 2006b; Dourado et al., 2009; Werle et al., 2014a). Alguns modelos permitem o acúmulo de temperatura acima de valor limite mínimo (temperatura base) e em outros, acima de conteúdo de água no solo, limiar necessário para a germinação das sementes (Werle et al., 2014a). Os modelos TH melhoraram a precisão das previsões do modelo TT, em locais onde ocorrem períodos de déficit hídrico (Leguizamon et al., 2005).

O uso de TT nos modelos de emergência tornou-se bem sucedido com a constatação de que a emergência pode ser representada por uma simples curva cumulativa sigmoidal (Gompertz, Weibull), ou funções logísticas nas quais o TT serve como variável independente (Brown e Mayer, 1988; Forcela, 2000). No entanto, o modelo não faz distinção de dormência, germinação e crescimento pré-emergência e, o processo de modelagem é iniciado em uma data fixa, independentemente de práticas de cultivo (Colbach et al., 2005).

Nos modelos mecanicistas a incorporação de variáveis fisiológicas e a divisão em subprocessos, tais como dormência, germinação e crescimento pré-emergência, bem como os efeitos ambientais sobre esses processos e a influência das práticas de manejo, tornam a modelagem mais complexa (Forcela, 2000; Grundy et al., 2003). Esses modelos necessitam de grandes e complexos conjuntos de entrada de dados que nem sempre são completas e confiáveis (Colbach et al., 2006). Para evitar o excesso de parametrização, a decomposição em subprocessos deve ser restrita aos aspectos que interagem com sistemas de cultivo, processos biológicos e efeitos ambientais (Colbach et al., 2006).

Segundo Grundy (2003) e Dourado et al. (2009), ambas as abordagens tem vantagens e desvantagens no que se refere à prática de predição da emergência, sendo que os modelos mecanicistas permitem melhor compreensão sobre a emergência de plantas daninhas, mas os modelos empíricos podem oferecer a simplicidade e a flexibilidade necessária para a tomada de decisão sobre o controle de plantas daninhas.

Para realização da modelagem é necessário conduzir experimentos a campo, no mínimo durante dois anos para monitorar a emergência semanal de plantas daninhas em função do ciclo de desenvolvimento e as covariáveis temperatura e potencial de água no solo a profundidade mínima de dois centímetros, juntamente com propriedades do solo (areia, silte, argila, matéria orgânica e $\mathrm{pH}$ ), sendo que a inclusão de outras variáveis vai depender da complexidade do modelo.

A obtenção dos parâmetros a campo pode ser determinada de várias formas, como o monitoramento por datalogger (Roman et al., 2000; Masin et al., 2012, 2014), estações meteorológicas (Roman et al., 2000; Spokas e Forcella, 2009; Werle et al., 2014a) e por informações de satélites. Entretanto, a maneira de obtenção de cada parâmetro necessita processos de correlações e calibração. Ainda, alguns parâmetros são derivados de laboratório, tais como limiares de umidade e temperatura (Werle et al., 2014a; Royo-Esnal et al., 2015). 


\section{Procedimento da modelagem utilizando o tempo hidrotérmico}

Os dados de emergência são convertidos de contagens semanais para emergência cumulativa, com base na emergência total de mudas (Masin et al., 2012; Werle et al., 2014a, 2014b). Da mesma forma, os dados de temperatura e umidade são utilizados para determinar o TT ou TH, de acordo com equação sugerida por Gummerson (1986):

$\mathrm{HT}=\sum(\theta \mathrm{H} \times \theta \mathrm{T})$

na qual: $\theta \mathrm{H}=1$, quando $\Psi>\Psi b$; caso contrário $\theta \mathrm{H}=0 ; \mathrm{e} \theta \mathrm{T}=\mathrm{T}-\mathrm{Tb}$ quando $\mathrm{T}>\mathrm{Tb}$, caso contrário $\theta \mathrm{T}=0 ; \Psi$ é o potencial médio diário da água na camada do solo; $\Psi$ b é o potencial de água base para emergência de plântulas; $T$ é a temperatura média diária do solo na profundidade aferida e Tb é a temperatura de base para a emergência das plântulas (Martinson et al., 2007; Royo-Esnal et al., 2010). Com esta fórmula, o TH só é acumulado quando as condições $\Psi$ e $\mathrm{T}$ foram superiores a $\Psi \mathrm{b}$ e $\mathrm{Tb}$. Então, a relação entre a emergência cumulativa é descrita pelo modelo de Gompertz e/ou Weibull:

Gompertz y=100exp $(-\mathrm{aexp}(-\mathrm{bHT}))$

onde: y é a porcentagem de emergência; $a$ está relacionada com um atraso HT antes do início da emergência; e, $b$ é relacionada à inclinação da curva.

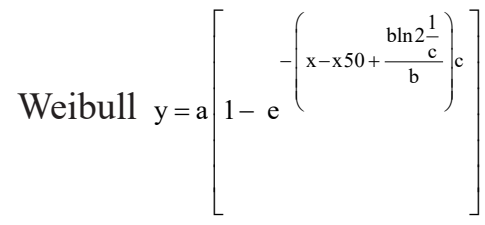

onde: $\mathrm{y}$ é a porcentagem de emergência; $\mathrm{x}$ é o tempo expresso como HT; e, $a, b, c$ e x 50 são constantes derivadas empiricamente: $a$, é a porcentagem máxima de emergência registrada; $b$ é a taxa de aumento; $c$ é um parâmetro de forma; e, x50 é o HT necessário para obter 50\% de emergência.

Os modelos desenvolvidos são validados com dados de emergência de diferentes locais e não do local da pesquisa (Royo-Esnal et al., 2015) e os valores reais da emergência com os valores estimados pelo modelo devem ser analisados pelo quadrado médio do erro (RMSEP) e/ou pelo critério de informação de Akaike (AIC) (Chantre et al., 2012):

$$
\mathrm{RMSE}=\sqrt{1 / \mathrm{n} \sum_{\mathrm{i}=1}^{\mathrm{n}}(\mathrm{Pi}-\mathrm{Oi})^{2}}
$$

no qual: $P i$ representa a porcentagem cumulativa prevista de emergência; $O i$ é a porcentagem cumulativa efetiva de emergência; e, $n$ é o número de observações (Mayer e Butler, 1993; Roman et al., 2000).

$$
\mathrm{AIC}=\log \left(\mathrm{RMSE}^{2}\right)+\frac{2 \mathrm{~m}^{\mathrm{d}}}{\mathrm{N}}
$$

Onde: m é o número de parâmetros do modelo; N é o número de observações; e, d é uma constante definida pelo usuário (Qi e Zhang, 2001). O RMSE e AIC fornecem uma medida da diferença típica entre os valores previstos e os reais em unidades de percentagem de concentração de plântulas, assim os menores valores de RMSE e AIC permitem a seleção do modelo pelo ajuste satisfatório aos dados (Chantre et al., 2012; Royo-Esnal et al., 2015).

\section{Impactos práticos}

Sistemas de cultivo que utilizam pouco ou nenhum herbicida necessitam de informações sobre o fluxo de emergência das plantas daninhas para planejar práticas culturais (sistema de cultivo, época de semeadura, ciclo da cultura, espaçamento e densidade) e, aqueles que dependem de estratégias químicas precisam dessas informações para planejar o melhor momento para aplicação de herbicidas (Buhler et al., 1997, Werle et al., 2014a). O conhecimento do fluxo de emergência das espécies do banco de sementes, em diferentes épocas do ano, pode servir para adequar práticas de manejo e racionalizar o uso de herbicidas, com base nas premissas do MIPD.

Resultados de pesquisa realizados na cultura do milho e soja utilizando modelo de emergência evidenciaram três padrões de emergência 
(precoce, médio e tardio) (Dourado et al., 2009; Werle et al., 2014a). As espécies Abutilon theophrasti, Ambrosia artemisiifolia, Chenopodium album, Datura stramonium, Datura ferox, Digitaria sanguinalis, Sorghum halepense, emergem no inicio do ciclo da cultura; Cyperus rotundus, Echinochloa Crus-galli, Setaria faberi, Setaria pumila, Setaria viridis, Solanum ptychanthum, Solanum nigrum Xanthium strumarium, são espécies com emergência intermediária, durante o ciclo da cultura; e, espécie como Amaranthus retroflexus, Amaranthus rudis, Ipomoea hederacea, Sonchus oleraceus e Sorghum bicolor, tem aparecimento tardio (Dourado et al., 2009; Werle et al., 2014a), porém, algumas espécies apresentam fluxo de emergência curto e outras prolongado (Werle et al., 2014a). Entretanto, o maior fluxo de emergência de plantas daninhas ocorre aproximadamente um mês após a semeadura (Masin et al., 2012) e, o mesmo modelo utilizado para prever emergência de plantas daninhas na cultura do milho pode ser utilizado para a cultura da soja (Masin et al., 2014).

Em cereais de inverno esses padrões de emergência não foram observados, mas em condições de inverno quente e úmido a emergência de planta daninha ocorre em períodos mais cedo e em fluxos menores, porém em invernos frios e secos a emergência ocorre no final do ciclo (Izquierdo et al., 2013; Royo-Esnal et al., 2012). Determinou-se que Conyza bonariensis exige acúmulo de $15 \mathrm{TH}$ para iniciar a emergência e até a sexta semana após a semeadura ocorre $95 \%$ da emergência, tanto no outono quanto na primavera (Zambrano-Navea et al., 2013).

Os resultados das pesquisas sobre a época e do tempo de emergência de plantas daninha, podem ser usadas para programar a época de semeadura e o manejo durante o ciclo da cultura. Assim, para as espécies que apresentam emergência precoce e em áreas com histórico de infestações pode-se realizar a aplicação localizada de herbicidas pré-emergentes. Por outro lado, para as espécies que apresentam emergência tardia na cultura pode-se eliminar a aplicação de herbicidas pré-emergentes com alta atividade residual no solo e utilizar herbicidas em pós-emergência, no momento do maior fluxo de emergência das plantas daninhas e dessa forma, reduzir os custos de produção e os impactos da aplicação de herbicidas no ambiente. No entanto, para espécies que apresentam fluxo prolongado pode-se optar por utilizar herbicidas em pós-emergência com efeito residual e assim eliminar uma ou duas aplicação de herbicidas em estádios avançadas da cultura. Portanto, o conhecimento básico sobre os padrões de emergência das principais espécies de plantas daninhas presentes na cultura é necessário para estabelecer planos de controle (Dourado et al., 2009).

O acompanhamento periódico das lavouras é importante e deve ser preconizado, com o intuito de selecionar a melhor alternativa de controle e apenas quando necessário, entretanto em extensas áreas isso é difícil e oneroso. Dessa forma, os dados de modelagem podem ser utilizados como parâmetros para monitorar o fluxo de emergência de plantas daninhas, através de informações climáticas de satélites e/ou estações meteorológicos. Com essas informações é possível georreferenciar áreas e confeccionar mapas mais rapidamente para aplicar herbicidas com precisão, quando e onde o nível de dano econômico é atingido (Santi et al., 2014).

Cabe ressaltar que as práticas de manejo e condições climáticas influenciam a dinâmica de emergência das espécies presentes no banco de sementes (Chauhan et al., 2006c), assim o desenvolvimento de modelos de emergência pode contribuir para aprimorar o MIPD, podendo ser considerado uma das melhores alternativas para contornar os problemas de resistência de plantas a herbicidas, visto que é possível reduzir a pressão de seleção e ainda realizar a aplicação nos estádios iniciais de desenvolvimento, onde as plantas são mais suscetíveis.

\section{Considerações finais}

A modelagem matemática do fluxo de emergência de plantas daninhas é uma ferramenta para a tomada de decisão no manejo de cultivos e deve ser utilizada para aprimorar práticas de 
manejo em diferentes épocas de semeadura. As respostas diferentes de cada espécie ao acúmulo de temperatura, umidade do solo, sistema de cultivo e o efeito aleatório do ano, propõe a necessidade de modelos mais complexos para a compreensão da emergência de plantas daninhas sobre a ecologia e biologia das espécies, enquanto modelos mais simples podem ser utilizados para apoiar a tomada de decisão sobre o controle de plantas daninhas.

\section{Referências}

Ali, H.H.; Tanveer, A.; Nadeem, M.A.; Asghar, H.N.; Javaid, M.M. Germination ecology of Rhynchosia capitata: an emerging summer weed in Asia. Planta Daninha, v.31, n.2, p.249-257, 2013.

Andrade, A.C.S.; Souza, A.F.; Ramos, F.N.; Pereira, T.S.; Cruz, A.P.M. Germinação de sementes de jenipapo: temperatura, substrato e morfologia do desenvolvimento pós-seminal. Pesquisa Agropecuária Brasileira, v.35, n.3, p.609-615, 2000.

Blanco, D.A.; Blanco, F.M.G. Efeito do manejo do solo na emergência de plantas daninhas anuais. Pesquisa Agropecuária Brasileira, v.26, n.2, p.215-220, 1991.

Blanco, F.M.G. Classificação e mecanismos de sobrevivência das plantas daninhas. In: Monqueiro, P.A. Aspectos da biologia e manejo das plantas daninhas. São Paulo: SBCPD, 2014. p.33-59.

Brown, R.F.; Mayer, D.G. Representing cumulative germination. 2. The use of the Weibull function and other empirically derived curves. Annals of Botany, v.61, p.127-138, 1988.

Buhler, D.d.; King, R.P.; Swinton, S.M.; Gunsolus, J.L.; Forcella, F. Field evaluation of a bioeconomical model for weed management in Soybean. Weed Science, v.45, n.1, p.158-165, 1997.

Calado, J.M.G.; Basch, G.; Carvalho, M. Weed emergence in autumn under temperate conditions. Planta Daninha, v.29, n.2, p.343-349, 2011.
Canossa, R.S.; Oliveira Junior, R.S.; Constantin, J.; Braccini, A.L.; Biffe, D.F.; Alonso, D.G.; Blainski, E. Temperatura e luz na germinação das sementes de apaga-fogo (Alternanthera tenella). Planta Daninha, v.26, n.4, p.745-750, 2008.

Carvalho, N.M.; Nakagawa, J. Sementes: ciência, tecnologia e produção. 4.ed. Jaboticabal: FUNEP, 2000. 588p.

Chantre, G.R.; Blanco, A.M.; Lodovichi, M.V.; Bandoni, A.J.; Sabbatini, M.R.; López, R.L.; et al. Modeling Avena fatua seedling emergence dynamics: an artificial neural network approach. Computers and Electronics in Agriculture, v.88, n.2, p.95102, 2012.

Chauhan, B.S.; Gill, G.; Preston, C. Factors affecting seed germination of annual sowthistle (Sonchus oleraceus) in southern Australia. Weed Science, v.54, n.5, p.854-860, $2006 a$.

Chauhan, B.S.; Gill, G.; Preston, C. Seedling recruitment pattern and depth of recruitment of 10 weed species in minimum tillage and no-till seeding systems. Weed Science, v.54, n.4, p.658-668, $2006 \mathrm{~b}$.

Chauhan, B.S.; Gill, G.; Preston, C. Influence of environmental factors on seed germination and seedling emergence of rigid ryegrass (Lolium rigidum). Weed Science, v.54, n.6, p.1004-1012, 2006c.

Colbach, N.; Dürr, C.; Roger-Estrade, J.; Caneill, J. How to model the effects of farming practices on weed emergence. Weed Research, v.45, n.1, p.2-17, 2005.

Colbach, N.; Dürr, C.; Roger-Estrade, J.; Chauvel, B. Alomysys: modelling black-grass (Alopecurus myosuroides Huds.) germination and emergence, in interaction with seed characteristics, tillage and soil climate: I. construction. European Journal of Agronomy, v.24, n.2, p.95-112, 2006.

Derakhshan, A.; Gherekhloo, J.; Vidal, R.A.; Prado, R. Quantitative description of the germination of Littleseed Canarygrass (Phalaris minor) in response to temperature. Weed Science, v.62, n.2, p.250257, 2014. 
Dourado, J.; Sousa, E.; Calha, I.M.; González-Andújar, J.L.; Fernández-Quintanilla, C. Predicting weed emergence in maize crops under two contrasting climatic conditions. Weed Research, v.49, n.3, p.251-260, 2009.

Forcella, F. Modelling seedling emergence. Field Crops Research, v.67, n.2, p.123-139, 2000.

Grundy,A.C. Predicting weed emergence: a review of approaches and future challenges. Weed Research, v.43, n.1, p.1-11, 2003.

Grundy, A.C.; Peters, N.C.B.; Rasmussen, I.A.; Hartmann, K.M.; Sattin, M.L.; Andersson, A.; et al. Emergence of Chenopodium album and Stellaria media of different origins under different climatic conditions. Weed Research, v.43, n.3, p.163-176, 2003.

Guimarães, M.A.; Dias, D.C.F.S.; Loureiro, M.E. Hidratação de sementes. Revista Trópica, v.2, n.1, p.31-39, 2008.

Gummerson, R.J. The effect of constant temperatures and osmotic potential on the germination of sugar beet. Journal of Experimental Botany, v.37, n.6, p.729741, 1986.

Guo, P.; Al-Khatib, K. Temperature effects on germination and growth of redroot pigweed (Amaranthus retroflexus), Palmer amaranth (A. palmeri), and common waterhemp (A. rudis). Weed Science, v.51, n.6, p.869-875, 2003.

Izquierdo, J.; Bastida, F.; Lezaún, J.M.; Sanchez Del Arco, M.J.; Gonzalez-Andujar, J.L. Development and evaluation of a model for predicting Lolium rigidum emergence in winter cereal crops in the Mediterranean area. Weed Research, v.53, n.4, p.269-278, 2013.

Klein, A.; Felippe, G.M. Efeito da luz sobre a germinação de sementes de ervas invasoras. Pesquisa Agropecuária Brasileira, v.26, n.7, p.955-966, 1991.

Koger, C.H.; Reddy, K.N.; Poston, D.H. Factors affecting seed germination, seedling emergence and survival of texasweed (Caperonia palustris). Weed Science, v.52, n.6, p.989-995, 2004.
Leguizamon, E.S.; Fernandez-Quintanilla, C.; Barroso, J.; Gonzalez-Andujar, J.L. Using thermal and hydrothermal time to model seedling emergence of Avena sterilis ssp. Ludoviciana in Spain. Weed Research, v.45, n.2, p.149-156, 2005.

Martins, C.C.; Martins, D.; Negrisoli, E.; Stanguerlim, H. Comportamento germinativo de sementes de leiteiro (Peschiera fuchsiaefolia): efeito da temperatura e luz. Planta Daninha, v.18, n.1, p.85-91, 2000.

Martinson, K.; Durgan, B.; Forcella, F.; Wiersma, J.; Spokas, K.; Archer, D. An emergence model for wild oat (Avena fatua). Weed Science, v.55, n.6, p.584-591, 2007.

Masin, R.; Loddo, D.; Benvenuti, S.; Otto, S.; Zanin, G. Modeling Weed Emergence in Italian Maize Fields. Weed Science, v.60, n.2, p.254-259, 2012.

Masin, R.; Loddo, D.; Gasparini, V.; Otto, S.; Zanin, G. Evaluation of Weed Emergence Model AlertInf for Maize in Soybean. Weed Science, v.62, n.2, p.360-369, 2014.

Mayer, D.G.; Butler, D.G. Statistical validation. Ecological Modelling, v.68, n.1, p.21-32, 1993.

Mondo, V.H.V.; Carvalho, S.J.P.; Dias, A.C.R.; Marcos Filho, J. Efeitos da luz e temperatura na germinação de sementes de quatro espécies de plantas daninhas do gênero Digitaria. Revista Brasileira de Sementes, v.32, n.1, p.131-137, 2010.

Myers, M.W.; Curran, W.S.; Vangessel, M.J.; Calvin, D.D.; Mortensen, D.A.; Majek, B.A.; et al. Predicting weed emergence for eight annual species in the northeastern United States. Weed Science, v.52, n.6, p.913-919, 2004.

Orzari, I.; Monquero, P.A.; Reis, F.C.; Sabbag, R.S.; Hirata, A.C.S. Germinação de espécies da família Convolvulaceae sob diferentes condições de luz, temperatura e profundidade de semeadura. Planta Daninha, v.31, n.1, p.53-61, 2013.

Popinigis, F. Fisiologia da semente. 2.ed. Brasília, DF: AGIPLAN, 1985, 289 p.

Qi, M.; Zhang, G.P. An investigation of model selection criteria for neural network time series 
forecasting. European Journal of Operational Research, v.132, n.3, p.666-680, 2001.

Roman, E.S.; Murphy, S.D.; Swanton, C.J. Simulation of Chenopodium album seedling emergence. Weed Science, v.48, n.2, p.217-224, 2000.

Royo-Esnal, A.; Gesch, R.W.; Forcella, F.; Torra, J.; Recasens, J.; Necajeva, J. The role of light in the emergence of weeds: Using Camelina microcarpa as an example. PLoS One, v.10, n.12, p.1-12, 2015.

Royo-Esnal, A.; Torra, J.; Conesa, J.A.; Forcella, F.; Recasens, J. Modeling the emergence of three arable bedstraw (Galium) species. Weed Science, v.58, n.1, p.10-15, 2010.

Royo-Esnal, A.; Torra, J.; Conesa, J.A.; Recasens, J. Emergence and early growth of Galium aparine and Galium spurium. Weed Research, v.52, n.5, p.458-466, 2012.

Sadeghloo, A.; Asghari, J.; Ghaderi-Far, F. Seed germination and seedling emergence of Velvetleaf (Abutilon theophrasti) and Barnyardgrass (Echinochloa crus-galli). Planta Daninha, v.31, n.2, p.259-266, 2013.

Salvador, F.L.; Victoria Filho, R.; Alves, A.S.R.; Simoni, F.; San Martin, H.A.M. Efeito da luz e da quebra de dormência na germinação de sementes de espécies de plantas daninhas. Planta Daninha, v.25, n.2, p.303-308, 2007.

Santi, A.L.; Bona, S.D.; Lamego, F.P.; Basso, C.J.; Eitelwein, M.T.; Cherubin, M.R.; et al. Phytosociological variability of weeds in soybean field. Planta Daninha, v.32, n.1, p.39-49, 2014.

Souza Filho, A.P.S.; Alves, S.M.; Figueiredo, F.J.C.; Dutra, S. Germinação de sementes de plantas daninhas de pastagens cultivadas: Mimosa pudica e Ipomoea asarifolia. Planta Daninha, v.19, n.1, p.23-31, 2001.

Spokas, K.; Forcella, F. Software tools for weed seed germination modeling. Weed Science, v.57, n.2, p.216-227, 2009.
Vázquez-Yanes, C.; Orozco-Segovia, A. Ecological significance of light controlled seed germination in two contrasting tropical habitats. Oecologia, v.83, n.2, p.171-175, 1990.

Vidal, R.A.; Kalsing, A.; Goulart, I.C.G.R.; Lamego, F.P.; Christoffoleti, P.J. Impacto da temperatura, irradiância e profundidade das sementes na emergência e germinação de Conyza bonariensis e Conyza canadensis resistentes ao glyphosate. Planta Daninha, v.25, n.2, p.309-315, 2007.

Voll, E.; Karam, D.; Gazziero, D.L.P. Dinâmica de populações de capim-colchão (Digitara horizontalis Willd.) sob manejos de solo e de herbicidas. Pesquisa Agropecuária Brasileira, v.32, n.4, p.373-378, $1997 \mathrm{a}$.

Voll, E.; Karam, D.; Gazziero, D.L.P. Dinâmica de populações de trapoeraba (Commelina benghalensis L.) sob manejos de solo e de herbicidas. Pesquisa Agropecuária Brasileira, v.32, n.6, p.571-578, 1997b.

Werle, R.; Sandell, D.L.; Buhler, D.D.; Hartzler, R.G.; Lindquist, J.L. Predicting emergence of 23 summer annual weed species. Weed Science, v.62, n.2, p.267-279, 2014a.

Werle, R.; Bernards, M.L.; Arkebauer, T.J.; Lindquist, J.L. Environmental triggers of winter annual weed emergence in the Midwestern United States. Weed Science, v.62, n.1, p.83-96, 2014b.

Yamashita, O.M.; Guimarães, S.C. Germinação de sementes de Conyza canadensis e Conyza bonariensis em diferentes condições de temperatura e luminosidade. Planta Daninha, v.29, n.2, p.333342, 2011.

Zambrano-Navea, C.; Bastida, F.; Gonzalez-Andujar, J.L. A hydrothermal seedling emergence model for Conyza bonariensis. Weed Research, v.53, n.4, p.213-220, 2013. 